\title{
Experience of applying cytoreductive surgery and hyperthermic intraperitoneal chemotherapy for ovarian teratoma with malignant transformation and peritoneal dissemination
}

This article was published in the following Dove Medical Press journal:

Therapeutics and Clinical Risk Management

\author{
Hsin-Hsien $\mathrm{Yu}^{1,2}$ \\ Yutaka Yonemura ${ }^{3,4}$ \\ Mao-Chih Hsieh ${ }^{2,5}$ \\ Chang-Yun $\mathrm{Lu}^{2}$ \\ Szu-Yuan $\mathrm{Wu}^{6,7}$ \\ Yan-Shen Shan ${ }^{1,8}$
}

'Institute of Clinical Medicine, College of Medicine, National Cheng Kung University, Tainan, Taiwan; ${ }^{2}$ Division of General Surgery, Department of Surgery, Wan Fang Hospital, Taipei Medical University, Taipei, Taiwan; ${ }^{3}$ Peritoneal Dissemination Center, Kishiwada Tokushukai Hospital, Kishiwada, Japan; ${ }^{4}$ Department of Surgery, Kusatsu General Hospital, Kusatsu, Shiga, Japan; ${ }^{5}$ Department of Surgery, School of Medicine, College of Medicine, Taipei Medical University, Taipei, Taiwan; ${ }^{6}$ Department of Radiation Oncology, Wan Fang Hospital, Taipei Medical University, Taipei, Taiwan; ${ }^{7}$ Department of Internal Medicine, School of Medicine, College of Medicine, Taipei Medical University, Taipei, Taiwan; ${ }^{8}$ Department of Surgery, National Cheng Kung University Hospital, College of Medicine, National Cheng Kung University, Tainan, Taiwan

Correspondence: Yan-Shen Shan Department of Surgery, National Cheng Kung University Hospital, Institute of Clinical Medicine, College of Medicine, National Cheng Kung University, Tainan, Taiwan

Tel +88662353535 ext 3105

Fax +88662758781

Email ysshan@mail.ncku.edu.tw
Objectives: The prognosis of ovarian teratoma with malignant transformation and peritoneal dissemination (PD) is poor. This condition is rare but associated with a high recurrence rate even after aggressive debulking surgery and adjuvant chemotherapy. In the present paper, we describe our experience of using cytoreductive surgery and hyperthermic intraperitoneal chemotherapy (CRS-HIPEC) for this condition.

Methods: The data of ten female patients having ovarian teratoma with malignant transformation and PD between June 2007 and June 2017 were collected and reviewed retrospectively. CRSHIPEC was performed according to the standard protocol. Patient characteristics, pathological reports, tumor markers, perioperative operative parameters, postoperative events, and disease status during the follow-up period were recorded.

Results: The primary ovarian neoplasms were pure mature cystic teratoma with malignant transformation ( $\mathrm{n}=6$, including 5 of mucinous adenocarcinoma), mixed germ cell tumor with mature cystic teratoma and yolk sac tumor (YST) $(n=1)$, pure immature teratoma $(n=1)$, immature teratoma with growing teratoma syndrome (GTS) $(n=1)$, and immature teratoma mixed YST with GTS $(n=1)$. The mean levels of tumor markers, including carcinoembryonic antigen, cancer antigen 19-9 (CA19-9), and CA125, were markedly elevated. The recurrence rate was $10 \%$. The median and mean disease-free survival (DFS) after CRS-HIPEC were 22.3 and 36.2 months, respectively, and the 5-year DFS rate is $88 \%$.

Conclusion: CRS-HIPEC is a safe therapeutic option for reducing the recurrence rate in selected patients with PD originating from ovarian teratoma with malignant transformation.

Keywords: ovarian teratoma, malignant transformation, peritoneal dissemination, cytoreductive surgery, hyperthermic intraperitoneal chemotherapy

\section{Introduction}

Ovarian germ cell tumors (GCTs) originate from primordial germ cells and account for approximately $30 \%$ of all ovarian neoplasms. Malignant ovarian GCTs are an uncommon type of ovarian cancer, with incidence rates of $4.2 \%$ in Asia, 2.5\% in Oceania, $2.0 \%$ in North America, and $1.3 \%$ in Europe. ${ }^{1}$ Malignant ovarian GCTs generally occur in younger women, with an incidence rate of $75 \%$ in women aged less than 30 years. These GCTs account for two-thirds of ovarian cancers in the first two decades of life. According to the WHO's histological classification, the most frequent type of "malignant" ovarian GCT is from teratoma, which includes mature teratoma, immature teratoma, and monodermal teratoma (eg, struma ovarii, carcinoid tumor, neuroectodermal tumor, and sebaceous tumor). ${ }^{2}$ 
Most mature cystic teratomas are benign and can be managed using laparoscopic oophorectomy or ovarian fertility-sparing cystectomy in younger women. However, "malignant transformation" is rare but it can occur in $1 \%-2 \%$ of cases, particularly in the sixth to seventh decades of life. Not only for mature cystic teratoma with malignant transformation, immature cystic teratoma also has malignant potential and occasionally enlarges rapidly, with development of abdominal pain. For nondysgerminomatous GCTs, unilateral salpingo-oophorectomy with full staging is an appropriate surgical treatment, and adjuvant chemotherapy with platinum-based regimens is also considered for patients with advanced-stage tumors.

Although patients with malignant ovarian GCTs typically exhibit higher survival rates than those with epithelial ovarian cancers, the prognosis of malignant ovarian GCTs is still low. For example, Ghaemmaghami et $\mathrm{al}^{3}$ reported a median disease-free survival (DFS) of 40 months, median overall survival of 50 months, and 5-year survival rate of $39 \%$. The prognosis of mature cystic teratoma with malignant transformation is also poor. Most patients with this disease die within a year, and only a few patients have been reported to survive for an exceptionally long time. ${ }^{4}$ Similar to other intra-abdominal cancers, malignant ovarian teratomas with peritoneal dissemination (PD) are associated with a worse prognosis. McKenny et $\mathrm{al}^{5}$ reported nine cases of malignant ovarian teratoma with pseudomyxoma peritonei (PMP), including six cases that were available for follow-up. One patient died of the disease at 6 months. Further analysis of the detailed outcome revealed a median DFS of 23.5 months (mean DFS: 23.8 months) and a high recurrence rate of $50 \%$. The 5-year DFS rate was $40 \%$. These observations imply that a more aggressive strategy is necessary for treating ovarian teratoma with malignant transformation and PD.

Peritoneal carcinomatosis (PC) is considered an untreatable disease and is associated with a short life expectancy. However, the development of a comprehensive treatment strategy, namely cytoreductive surgery combined with hyperthermic intraperitoneal chemotherapy (CRS-HIPEC), has improved the survival of patients with this disease since the 1980s. Numerous studies have demonstrated that treatment with CRS-HIPEC significantly improves the survival and prognosis of patients with PC originating from appendiceal cancer, colorectal cancer, malignant peritoneal mesothelioma, gastric cancer, PMP, and ovarian cancer. ${ }^{6-10}$ In the present paper, we describe our experience of managing ovarian teratoma with malignant transformation and PD by applying CRS-HIPEC. To the best of our knowledge, it is also the first case series that has applied CRS-HIPEC to treat patients with this disease.

\section{Materials and methods Patient characteristics}

This study was conducted using a prospectively maintained database of patients receiving CRS-HIPEC for peritoneal surface malignancy at the Peritoneal Dissemination Centers of Kishiwada Tokushukai Hospital and Kusatsu General Hospital. The data of 10 female patients who had ovarian teratoma with malignant transformation to PD between June 2007 and June 2017 were collected and reviewed retrospectively. The study was conducted in accordance with the principles of the Declaration of Helsinki and approved by the institutional review boards of Kishiwada Tokushukai Hospital (Japan) and Kusatsu General Hospital (Japan). Written informed consent was obtained from all the patients at the time of hospital admission as a part of routine assessment before surgery, anesthesia and data collection. The prior surgical score (PSS), which quantifies the extent of any previous surgery before definitive CRS-HIPEC, was also recorded. ${ }^{11}$ All patients received computed tomography, magnetic resonance imaging, positron emission tomography or colonoscopy to rule out tumors originated from appendix or colorectum. The pathological review for correct diagnosis was all performed by the pathologists who were familiar to gynecological peritoneal surface malignancies.

\section{CRS-HIPEC}

CRS combined with HIPEC was performed according to the standard protocol of our institution. To determine the peritoneal cancer index (PCI) the dissemination status of the intra-abdominal malignancy was evaluated during exploration, following the principles described by Harmon. ${ }^{11}$ First, extensive intraoperative peritoneal lavage (EIPL) was performed 10 times by using $1 \mathrm{~L}$ of saline each time (total $=10 \mathrm{~L}$ ). CRS combined with peritonectomy was then performed to remove all macroscopically visible tumors and all intra-abdominal organs affected by the malignancy. EIPL was then repeated again. This was followed by the administration of HIPEC for 40 minutes by using the open technique at temperatures of $42.5^{\circ} \mathrm{C}-43.5^{\circ} \mathrm{C}$. The chemotherapeutic drugs used for HIPEC were docetaxel $\left(30 \mathrm{mg} / \mathrm{m}^{2}\right)$ plus cisplatin $\left(30-50 \mathrm{mg} / \mathrm{m}^{2}\right)$.

\section{Postoperative course and follow-up}

Postoperative completeness of cytoreduction (CC) scores was determined to evaluate the extent of CRS following 
the criteria described by Harmon. ${ }^{11}$ Notably, CC scores of $0,1,2$, and 3 indicated that there was no residual tumor, a $<2.5 \mathrm{~mm}$ residual tumor, a $2.5 \mathrm{~mm}-2.5 \mathrm{~cm}$ residual tumor, and a $>2.5 \mathrm{~cm}$ residual tumor, respectively. Postoperative morbidity and mortality were graded according to the Clavien-Dindo classification system, and selected patients received adjuvant chemotherapy. All patients were routinely followed up at outpatient clinics. Their age, pathological reports, tumor markers, PCI, CC score, postoperative PCI, operation time and blood loss, hospitalization, morbidity, neoadjuvant and adjuvant chemotherapy, and disease status at last known clinical follow-up were recorded from patient charts.

\section{Statistical analysis}

DFS was recorded after the diagnosis of malignant ovarian teratoma with PC and after CRS-HIPEC. The endpoint in this study was evidence of disease recurrence, last follow-up, or death. Because all of our patients received CRS-HIPEC, we compared the survival curve of our patients with that of the six patients with the same diseases who were treated with the current standard strategy in a study by McKenny et al. ${ }^{5}$ Survival comparison was made using the Kaplan-Meier estimator. The log-rank test was employed to identify significant differences and data were analyzed using SPSS version 20.0 (IBM Corporation, Armonk, NY, USA). $P<0.05$ was considered statistically significant.

\section{Results}

\section{Patient characteristics}

The clinical characteristics and detailed pathological findings of the 10 female patients having ovarian teratoma with malignant transformation and PD are listed in Tables 1 and 2. The mean age of these ten patients was 45.0 years (range: 20-69 years). Neoadjuvant systemic chemotherapy with cisplatin and with cisplatin plus docetaxel was administered to four patients and one patient, respectively. This one patient also received neoadjuvant intraperitoneal chemotherapy with cisplatin plus docetaxel as a part of bidirectional intraperitoneal and systemic induction chemotherapy. The mean levels of tumor markers, including carcinoembryonic antigen (CEA), cancer antigen 19-9 (CA19-9), and CA125, were markedly elevated. The typical presentation of PMP was found in eight patients, but was absent in the other two patients who were also diagnosed as having malignant immature teratoma with post-chemotherapy growing teratoma syndrome (GTS) (Table 2; patients 9 and 10). The pre-CRS ascites cytological examination revealed malignancy in two patients and suspected malignancy in another two patients.
Table I The epidemiologic and perioperative data of the ten female patients of ovarian teratoma with malignant transformation and peritoneal dissemination

\begin{tabular}{|c|c|}
\hline Characteristics & $\begin{array}{l}\text { All patients } \\
(\mathrm{N}=10)\end{array}$ \\
\hline Age, mean (range) (year) & $45(20-69)$ \\
\hline \multicolumn{2}{|l|}{ PSS, n } \\
\hline 0 & 2 \\
\hline I & 2 \\
\hline 2 & 6 \\
\hline Neoadjuvant systemic chemotherapy, $\mathrm{n}$ & 5 \\
\hline Neoadjuvant intraperitoneal chemotherapy, $n$ & I \\
\hline CAI25, mean (range) $(\mathrm{U} / \mathrm{mL})$ (normal 0-35 U/mL) & I45.5 (3.7-8|6.0) \\
\hline CEA, mean (range) $(\mathrm{ng} / \mathrm{mL})$ (normal $0-5 \mathrm{ng} / \mathrm{mL}$ ) & $93.9(1.2-787.0)$ \\
\hline CAI9-9, mean (range) $(\mathrm{U} / \mathrm{mL})$ (normal 0-37 U/mL) & $337.7(1.0-1,498.0)$ \\
\hline Presentation of PMP, $n$ & 8 \\
\hline \multicolumn{2}{|l|}{ Ascites cytology, n } \\
\hline No evidence of malignancy & 6 \\
\hline Suspected malignancy or benign atypia & 2 \\
\hline Malignancy & 2 \\
\hline \multicolumn{2}{|l|}{$\mathrm{PCl}$, mean (range) } \\
\hline Pre-op & $13.9(3-24)$ \\
\hline Post-op & $1.4(0-11)$ \\
\hline \multicolumn{2}{|l|}{ CC score, $n$} \\
\hline 0 & 7 \\
\hline I & 3 \\
\hline 2 & 0 \\
\hline 3 & 0 \\
\hline \multicolumn{2}{|l|}{ HIPEC regimens, $n$} \\
\hline Docetaxel plus cisplatin & 9 \\
\hline Docetaxel alone & I \\
\hline \multicolumn{2}{|l|}{ Ostomy procedures, $\mathrm{n}$} \\
\hline Colostomy & I \\
\hline Ileal conduit urinary diversion & I \\
\hline Cutaneous tube ureterostomy & I \\
\hline Operation time, mean (range) (minutes) & $267(135-392)$ \\
\hline Blood loss, mean (range) (mL) & $1,299(450-2,100)$ \\
\hline \multicolumn{2}{|l|}{ Histopathological types of malignancies, $n$} \\
\hline $\begin{array}{l}\text { Mature cystic teratoma malignant } \\
\text { transformation }\end{array}$ & 6 \\
\hline Mature cystic teratoma mixed YST & I \\
\hline Immature teratoma & I \\
\hline Immature teratoma with GTS & I \\
\hline Immature teratoma mixed YST with GTS & I \\
\hline \multicolumn{2}{|l|}{ Morbidity, n } \\
\hline Grade 0 & 7 \\
\hline Grade I-2 & 3 \\
\hline Grade 3-5 & 0 \\
\hline Admission days, mean (range) & $34.4(14-87)$ \\
\hline Adjuvant chemotherapy, $\mathrm{n}$ & 6 \\
\hline Recurrence of disease, $n$ & I \\
\hline \multicolumn{2}{|l|}{ Disease-free survival after diagnosis, months } \\
\hline Mean \pm SD (range) & $54.3 \pm 44.9(10-121.5)$ \\
\hline Median & 32.0 \\
\hline \multicolumn{2}{|l|}{ Disease-free survival after CRS-HIPEC, months } \\
\hline Mean \pm SD (range) & $36.2 \pm 41.9(1.5-114.0)$ \\
\hline Median & 22.3 \\
\hline
\end{tabular}

Abbreviations: CC, completeness of cytoreduction;CRS, cytoreductive surgery; GTS, growing teratoma syndrome; HIPEC, hyperthermic intraperitoneal chemotherapy; $\mathrm{PCl}$, peritoneal cancer index; PD, peritoneal dissemination; PMP, pseudomyxoma peritonei; PSS, prior surgical score; YST, yolk sac tumor. 
Table 2 Patient characteristics and pathological reviews

\begin{tabular}{|c|c|c|c|c|c|c|}
\hline Patient no. & Age & PMP & Ovary pathology & Appendix pathology & CK20/CK7 & DFS after CRS-HIPEC \\
\hline I & 65 & + & $\begin{array}{l}\text { Mature cystic teratoma with low } \\
\text { malignant potential mucinous neoplasm }\end{array}$ & Normal & $+1-$ & 108.0 \\
\hline 2 & 69 & + & $\begin{array}{l}\text { Mature cystic teratoma, low-grade } \\
\text { malignant mucinous adenocarcinoma }\end{array}$ & N/A & $-1+$ & 28.0 \\
\hline 3 & 59 & + & $\begin{array}{l}\text { Mature cystic teratoma, borderline } \\
\text { malignant mucinous adenocarcinoma }\end{array}$ & Normal & $+/+$ & 114.0 \\
\hline 4 & 20 & + & $\begin{array}{l}\text { Mature cystic teratoma, borderline } \\
\text { malignant mucinous adenocarcinoma }\end{array}$ & Normal & & 23.0 \\
\hline 5 & 30 & + & $\begin{array}{l}\text { Mature cystic teratoma, adenocarcinoma, } \\
\text { mixed Yolk sac tumor }\end{array}$ & Normal & $+1-$ & $6.5 *$ Recurrence \\
\hline 6 & 55 & + & Mature cystic teratoma, adenocarcinoma & Normal & & 1.5 \\
\hline 7 & 32 & + & Immature teratoma, mucinous adenocarcinoma & N/A & & 48.0 \\
\hline 8 & 47 & + & $\begin{array}{l}\text { Mature cystic teratoma, borderline } \\
\text { malignant mucinous adenocarcinoma }\end{array}$ & Normal & $+/+$ & 21.5 \\
\hline 9 & 46 & - & $\begin{array}{l}\text { Immature teratoma, post- } \mathrm{C} / \mathrm{T} \text { growth } \\
\text { teratoma syndrome }\end{array}$ & N/A & & 7.5 \\
\hline 10 & 27 & - & $\begin{array}{l}\text { Immature teratoma, mixed Yolk sac tumor, } \\
\text { post-C/T growth teratoma syndrome }\end{array}$ & N/A & & 3.5 \\
\hline
\end{tabular}

Abbreviations: CRS-HIPEC, cytoreductive surgery and hyperthermia intraperitoneal chemotherapy; DFS, disease-free survival; N/A, not available; PMP, pseudomyxoma peritonei.

\section{Surgical procedures - CRS-HIPEC}

The pre-CRS severity of peritoneal dissemination was evaluated using PCI. The mean PCI was 13.9 (range: 3-24). The surgical procedures for CRS included routine peritonectomy, omentectomy, bilateral salpingo-oophorectomy and total abdominal hysterectomy (if present), appendectomy, splenectomy, and cholecystectomy. In addition, low anterior resection for rectal invasion was performed in four patients (including one with sigmoid end colostomy) and left hemicolectomy was performed in one patient. Tumor invasion to the bladder and ureter was found in one patient; thus, ileal conduit urinary diversion was performed. Tumor invasion to the ureter was found in another patient, and cutaneous tube ureterostomy was thus performed. After CRS, the completeness of tumor clearance was determined using the $\mathrm{CC}$ score and post-PCI. A CC score of 0 was achieved in seven patients, and a CC score of 1 was achieved in three patients. The mean post-PCI was 1.4 (range: $0-11$, median: 0 ). These two indicators hinted at the appropriate completeness of tumor clearance in the patients. The chemotherapy regimens for HIPEC were docetaxel plus cisplatin in nine patients and docetaxel alone in one patient. The mean operation time was 267 minutes (range: 135-392 minutes) and the mean blood loss was 1,299 mL (range: 450-2,100 mL).

\section{Perioperative events and adjuvant chemotherapy}

Seven patients recovered uneventfully after CRS-HIPEC. According to the Clavien-Dindo classification system, grade 2 morbidity was detected in the remaining three patients. One of these patients experienced temporary renal insufficiency due to dehydration and wound poor healing, but recovered after adequate fluid supplementation and was discharged after 87 days of hospitalization. Another of these patients developed postoperative ileus and poor digestion but improved after fasting and nutrition support. A third of these patients developed postoperative fever that was successfully managed only with medical treatment. The mean hospitalization of the study individuals was 34 days (range: 14-87 days), after which all patients were followed up regularly at outpatient clinics. Six patients also received adjuvant chemotherapy with taxane-based regimens.

\section{Histopathology and immunohistochemical profiles}

The primary ovarian neoplasms in this study were pure mature cystic teratoma with malignant transformation $(\mathrm{n}=6$; including five of mucinous adenocarcinoma), mixed GCT with mature cystic teratoma and yolk sac tumor (YST) $(\mathrm{n}=1)$, pure immature teratoma $(\mathrm{n}=1)$, immature teratoma with GTS $(n=1)$, and immature teratoma mixed YST with GTS $(n=1)$. Of these ten malignant ovarian teratomas with peritoneal involvement, two were low-malignant potential mucinous neoplasm, three were low-grade mucinous adenocarcinoma, three were high-grade mucinous adenocarcinoma, and two were malignant potential immature teratoma with GTS (Table 2). We also determined the expression of cytokeratin 20 (CK20) and CK7 of tumors for revealing the 
immunophenotypes in seven of the study patients, and the immunophenotypes were CK20+/CK7- in four $(57.1 \%)$, CK20+/CK7+ in two (28.6\%), and CK20-/CK7+ in one patient $(14.3 \%)$. To clarify the origin of the primary tumor, we performed a pathological evaluation of the appendix after appendectomy. Seven appendectomy procedures were performed in the study population, and all pathological reports showed no neoplastic lesion or malignancy.

\section{Clinical follow-up and survival analysis}

Tumor recurrence was observed in only one patient. This 30-year-old female (patient 5) had malignant mixed GCT with a teratoma component and YST and received HIPEC with docetaxel alone, but showed disease recurrence over the pleural cavity at 6 months post-CRS. Thus, CRS of the intrathoracic lesion, followed by hyperthermic intrathoracic chemotherapy, was performed. Subsequently, she showed intrathoracic metastasis and intra-abdominal tumor recurrence, again after 6 months, and received CRS again. All pathological findings of the recurrent tumors revealed metastatic YST. Finally, after aggressive repeated CRS (3 times), no evidence of recurrence was noted in the following 15 months.

The recurrence rate was $10 \%$ ( 1 in 10 patients) and no patient mortality was noted during the study period. The median and mean DFS after the diagnosis of peritoneal dissemination were 32.0 and 54.3 months (range: 10-121.5 months) and the median and mean DFS after CRSHIPEC were 22.3 and 36.2 months (range: 1.5-114.0 months).

\section{Discussion}

Few studies have focused on the management of PD originating from ovarian teratoma with malignant transformation. Rim et al $^{12}$ reported 11 patients with malignant transformation arising from ovarian mature cystic teratoma who underwent bilateral salpingo-oophorectomy and total abdominal hysterectomy following adjuvant chemotherapy. In that report, only three patients were positive for ascites containing malignant cells or pelvic tumor implants, and their International Federation of Gynecology and Obstetrics (FIGO) stages were IC, IIB, and IIC. DFS was 8, 29, and 9 months for patients with FIGO IC, IIB, and IIC stages, respectively. The patient with a FIGO IC stage tumor died of intestinal obstruction at 8 months after surgery. The findings suggested that early detection and complete CRS are the only reliable methods for treating the advanced stage of this disease. Elsewhere, Hayes-Jordan et $\mathrm{al}^{13}$ firstly reported the application of CRS-HIPEC for treating a pediatric ovarian tumor. In their report, a 14-year-old girl with ovarian immature teratoma with peritoneal dissemination received CRS-HIPEC with a DFS of 44 months, and the author concluded that complete resection and HIPEC might be effective.

Most teratomas are benign, but rarely malignant transformation can occur. The rate of malignant transformation has been reported to be $1.7 \%$ for mature cystic teratoma, with a median age of 50.6 years. ${ }^{12}$ Malignant transformation to carcinoma may arise from any epithelial elements of the teratoma, and the most common form is transformation to squamous cell carcinoma (SCC), accounting for $70 \%$. Other transformed tumors originating from teratoma include adenocarcinoma (accounting for $7 \%$ of cases), basal cell carcinoma, sebaceous tumor, melanoma, sarcoma, and neuroendocrine tumor. The etiology of tumors other than $\mathrm{SCC}$ is also a poor prognostic factor. We discovered that all of our malignant ovarian mature cystic teratomas with peritoneal dissemination were of the intestinal type, including five adenocarcinomas and two intestinal-type low-malignant potential neoplasms. Intestinal-type mucinous neoplasms may exhibit a characteristic of easier dissemination in the abdominal cavity, which is a reasonable rationale for the application of HIPEC treatment.

Immunohistochemical analysis can be applied for the diagnosis of intestinal-type mucinous carcinoma arising from mature cystic teratoma, typically revealing positive staining for CK20 and Muc-2, but negative staining for CK7, Muc$5 \mathrm{AC}$, and Muc-6 in the adenocarcinomatous part. ${ }^{14}$ In the present study, the immunophenotypes of most patients were CK20+/CK7- (four patients, 57.1\%) and CK20+/CK7+ (two patients, $28.6 \%$ ); by contrast, only one patient presented with the immunophenotype of CK20-/CK7+. Moreover, another critical finding in the present study was the macroscopically and microscopically normal appendix, suggesting that the ovarian mucinous tumor was the primary tumor in the study population. Seven appendectomy procedures were performed in the study population, and all pathological reports showed no neoplastic lesion or malignancy.

The clinical usefulness of tumor markers in patients with malignant ovarian teratoma is unclear. The tumor markers for preoperative diagnosis and early detection of tumor recurrence should depend on the primary tumor components. Because the most common form of malignant ovarian teratoma is SCC, several studies have suggested that the SCC antigen level should be measured for diagnosis and follow-up. Indeed, in a case series of malignant cystic teratoma with a SCC component, Rim et al ${ }^{12}$ found elevated SCC antigen levels in $4(57 \%)$ of 7 patients. For nondysgerminomatous ovarian GCTs such as YST, choriocarcinoma, and some immature teratoma, alpha-fetoprotein 
and beta-human chorionic gonadotropin are useful tumor markers. In our case series of predominant intestinal-type malignant ovarian teratoma, we found marked elevation of CEA, CA19-9, and CA125, instead of the aforementioned markers. Though CA125 is more specific for ovary malignancy, it is not uncommon for simultaneous elevation of CEA, CA19-9, and CA125 in epithelial cancers at the stage of peritoneal carcinomatosis.

Malignant immature teratoma has been reported to be the second most common subtype of malignant ovarian GCTs. In one report, pure immature teratoma accounted for $35.6 \%$ $(449 / 1,262)$ of malignant ovarian GCT cases. ${ }^{15}$ Immature teratoma has malignant potential and occasionally enlarges rapidly. Adjuvant chemotherapy with cisplatinum-based regimens, typically with bleomycin, etoposide, and cisplatin, is still considered the standard for treating immature teratomas with stages greater than grade I or stage IA. ${ }^{16}$ For more advanced-stage immature teratomas, CRS followed by adjuvant chemotherapy is promoted as the optimal treatment. However, some patients may develop chemoresistance. Colombo et $\mathrm{al}^{17}$ reported that adjuvant chemotherapy with platinum-based regimens with bleomycin, etoposide and cisplatin (BEP) regimen were widely used, but taxane-based regimens could also be considered as second line treatment. Rezk et al ${ }^{18}$ reviewed the literature about malignant immature teratoma recurrence after primary surgery and those refractory to systemic chemotherapy, and reported that aggressive debulking surgery (ie, second salvage surgery) may improve patient outcome. The recurrence of pure mature teratoma may occur after chemotherapy for immature teratoma; this phenomenon is called GTS. In the present study, GTS was noted in two patients with intra-abdominal disseminated immature teratoma. Elsewhere, Bentivegna et $\mathrm{al}^{19}$ reviewed 38 patients with immature teratoma who developed GTS and recommended aggressive debulking surgery to achieve a favorable overall prognosis. However, the recurrence rate was still high (26\%, 10 in 38 cases).

For managing early-stage malignant ovarian teratoma, fertility-sparing surgery with comprehensive surgical staging is currently recommended. ${ }^{20}$ Billmire et $\mathrm{al}^{21}$ proposed new guidelines, including cytologic examination of ascites; excision and biopsy of omental, peritoneal, and other intra-abdominal nodules; palpation and biopsy of enlarged retroperitoneal lymph nodes; and resection of the tumorcontaining ovary. Most patients with malignant ovarian teratoma can be cured; however, some patients in the advanced stages of the disease may experience tumor recurrence. Because of the rarity of relapse in this population, no standard treatment approach has been established. Although aggressive debulking and adjuvant chemotherapy improved the overall survival of patients with malignant ovarian GCTs, the recurrence rate remains high. The rationale for applying CRS-HIPEC to patients with advanced-stage malignant ovarian teratoma was extrapolated from experience with epithelial ovarian cancer and other peritoneal surface malignancies. For tumors disseminated in the intra-abdominal cavity, the direct cytotoxic effect of HIPEC may eradicate invisible cancer nodules or cells after complete CRS. This rationale has been widely applied to manage PC originating from ovarian cancer, colorectal cancer, gastric cancer, malignant peritoneal mesothelioma, and appendiceal cancer with PMP. In the current study, we applied CRS-HIPEC as a new strategy for patients with malignant ovarian teratoma. Our study has the largest sample size in the literature to date. The results show a low recurrence rate $(10 \%)$ and no mortality during our study period; we also found a high average DFS of 36.15 months and a high 5-year DFS rate of $88 \%$. Because all of our patients received CRS-HIPEC (HIPEC group), we tried to compare the survival curve of our patients with that of the six patients with the same diseases who were treated with the current standard strategy in the study by McKenny et $\mathrm{al}^{5}$ (standard group). In the standard group, three patients showed disease recurrence after surgery, and one patient died of the disease. Additionally, the median and mean DFS after surgery were 23.5 and 23.8 months (range: 0-61 mo). The HIPEC group showed a higher 5-year DFS rate (88\%) than the standard group (40\%). The DFS curve also showed a trend of superior prognosis for the HIPEC group compared with that for the standard group (Figure 1). However, because of the small sample size, this finding did not reach statistical significance $(P=0.119)$.

Using CRS-HIPEC for treatment of peritoneal metastases from intraabdominal cancers has evolved over the last 2 decades. As the surgical techniques have improved and the HIPEC procedures standardized, the perioperative morbidity and mortality have been reduced markedly. Smeenk et $\mathrm{al}^{22}$ showed that perioperative mortality could be diminished by $50 \%$ and overall major morbidity was reduced from $71 \%$ between 1996 and 1998 to 34\% between 2003 and 2006 . Currently, major morbidity rates range from $25 \%$ to $35 \%$ and mortality from $1.5 \%$ to $12 \%{ }^{23}$ Moran $^{24}$ also reviewed the learning curve in this complex surgical procedure and showed that decision-making process and technical factors were critical. The most difficult decision was case selection. This implicated the urgent need for consensus about the indications of applying CRS-HIPEC. The main technical 


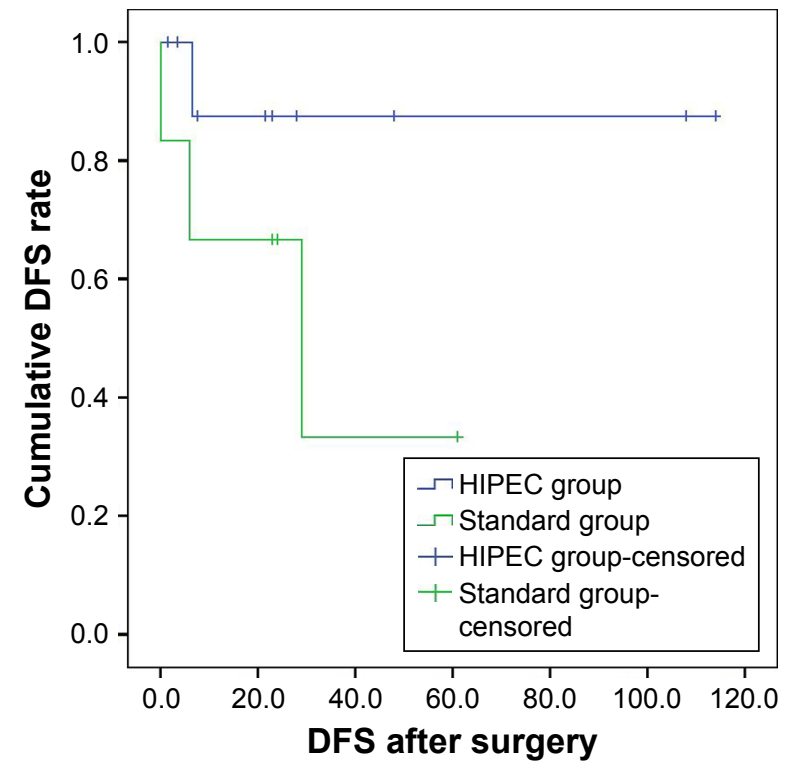

Figure I Comparison of survival curve of HIPEC group vs non-HIPEC standard treatment group; The HIPEC group showed a higher 5-year DFS rate (88\%) than the standard group (40\%) $(P=0.119)$.

Abbreviations: HIPEC, hyperthermic intraperitoneal chemotherapy; DFS, diseasefree survival.

factors that formed a significant part of the learning curve were anastomotic failure and postoperative hemorrhage. The application of proximal stomal diversion and meticulous attention to hemostasis before abdominal closure have been advocated to prevent anastomotic leakage and postoperative bleeding. Teamwork can also facilitate training and minimize the learning curve. Canda et $\mathrm{al}^{25}$ reviewed the toxicities after CRS-HIPEC and observed toxicity in $25.4 \%$ of patients, nephrotoxicity in $18.6 \%$, and hematological toxicity in $13.6 \%$. They concluded that prolonged operation times resulted in higher complication and/or toxicity rates, but outcomes were still acceptable for the treatment of peritoneal surface malignancies.

The present study results are unavoidably limited by the small sample and the short follow-up period in some cases. Further investigation and a longer observation period are necessary to verify the prognostic role of HIPEC in patients with malignant teratoma.

\section{Conclusion}

Our data showed that CRS-HIPEC is a safe therapeutic option for reducing the recurrence rate in selected patients with ovarian teratoma with malignant transformation and PD.

\section{Disclosure}

The authors report no conflicts of interest in this work.

\section{References}

1. Matz M, Coleman MP, Sant M, et al. The histology of ovarian cancer: worldwide distribution and implications for international survival comparisons (CONCORD-2). Gynecol Oncol. 2017;144(2):405-413.

2. Tavassoli FA, Devilee P, editors. WHO Classification of tumours. Pathology and Genetics of Tumours of the Breast and Female Genital Organs. 3rd ed. Lyon: IARC Press; 2003.

3. Ghaemmaghami F, Hasanzadeh M, Karimi Zarchi M, Fallahi A. Nondysgerminomatous ovarian tumors: clinical characteristics, treatment, and outcome. A case-controlled study. Int J Surg. 2008;6(5):382-386.

4. Ueda G, Fujita M, Ogawa H, Sawada M, Inoue M, Tanizawa O. Adenocarcinoma in a benign cystic teratoma of the ovary: report of a case with a long survival period. Gynecol Oncol. 1993;48(2):259-263.

5. McKenney JK, Soslow RA, Longacre TA. Ovarian mature teratomas with mucinous epithelial neoplasms: morphologic heterogeneity and association with pseudomyxoma peritonei. Am J Surg Pathol. 2008; 32(5):645-655.

6. Glehen O, Kwiatkowski F, Sugarbaker PH, et al. Cytoreductive surgery combined with perioperative intraperitoneal chemotherapy for the management of peritoneal carcinomatosis from colorectal cancer: a multi-institutional study. J Clin Oncol. 2004;22(16):3284-3292.

7. Yan TD, Deraco M, Baratti D, et al. Cytoreductive surgery and hyperthermic intraperitoneal chemotherapy for malignant peritoneal mesothelioma: multi-institutional experience. J Clin Oncol. 2009;27(36): 6237-6242.

8. Canbay E, Mizumoto A, Ichinose M, et al. Outcome data of patients with peritoneal carcinomatosis from gastric origin treated by a strategy of bidirectional chemotherapy prior to cytoreductive surgery and hyperthermic intraperitoneal chemotherapy in a single specialized center in Japan. Ann Surg Oncol. 2014;21(4):1147-1152.

9. Chua TC, Moran BJ, Sugarbaker PH, et al. Early- and long-term outcome data of patients with pseudomyxoma peritonei from appendiceal origin treated by a strategy of cytoreductive surgery and hyperthermic intraperitoneal chemotherapy. J Clin Oncol. 2012;30(20):2449-2456.

10. Bakrin N, Cotte E, Golfier F, et al. Cytoreductive surgery and hyperthermic intraperitoneal chemotherapy (HIPEC) for persistent and recurrent advanced ovarian carcinoma: a multicenter, prospective study of 246 patients. Ann Surg Oncol. 2012;19(13):4052-4058.

11. Harmon RL, Sugarbaker PH. Prognostic indicators in peritoneal carcinomatosis from gastrointestinal cancer. Int Semin Surg Oncol. 2005; 2(1):3-10

12. Rim SY, Kim SM, Choi HS. Malignant transformation of ovarian mature cystic teratoma. Int J Gynecol Cancer. 2006;16(1):140-144.

13. Hayes-Jordan A, Lopez C, Green HL, Xiao LC, Huh W, Herzog CE. Cytoreductive surgery (CRS) and hyperthermic intraperitoneal chemotherapy (HIPEC) in pediatric ovarian tumors: a novel treatment approach. Pediatr Surg Int. 2016;32(1):71-73.

14. Takai M, Kanemura M, Kawaguchi H, et al. Mucinous adenocarcinoma of the intestinal type arising from mature cystic teratoma of the ovary: a rare case report and review of the literature. J Ovarian Res. 2012;5(1):41.

15. Smith HO, Berwick M, Verschraegen CF, et al. Incidence and survival rates for female malignant germ cell tumors. Obstet Gynecol. 2006; 107(5):1075-1085.

16. Alwazzan AB, Popowich S, Dean E, Robinson C, Lotocki R, Altman AD. Pure immature teratoma of the ovary in adults. Int J Gynecol Cancer. 2015;25(9):1616-1622.

17. Colombo N, Peiretti M, Garbi A, et al. Non-epithelial ovarian cancer: ESMO clinical practice guidelines for diagnosis, treatment and follow-up. Ann Oncol. 2012;23(suppl 7):vii20-vii26.

18. Rezk Y, Sheinfeld J, Chi DS. Prolonged survival following salvage surgery for chemorefractory ovarian immature teratoma: a case report and review of the literature. Gynecol Oncol. 2005;96(3):883-887.

19. Bentivegna E, Azaïs H, Uzan C, et al. Surgical outcomes after debulking surgery for intraabdominal ovarian growing teratoma syndrome: analysis of 38 cases. Ann Surg Oncol. 2015;22(S3):964-970.

20. Gershenson DM. Management of ovarian germ cell tumors. J Clin Oncol. 2007;25(20):2938-2943. 
21. Billmire D, Vinocur C, Rescorla F, et al. Outcome and staging evaluation in malignant germ cell tumors of the ovary in children and adolescents: an intergroup study. J Pediatr Surg. 2004;39(3):424-429.

22. Smeenk RM, Verwaal VJ, Zoetmulder FA. Learning curve of combined modality treatment in peritoneal surface disease. Br J Surg. 2007;94(11): 1408-1414.

23. Verwaal VJ, van Tinteren H, Ruth SV, Zoetmulder FA. Toxicity of cytoreductive surgery and hyperthermic intra-peritoneal chemotherapy. J Surg Oncol. 2004;85(2):61-67.
24. Moran BJ. Decision-making and technical factors account for the learning curve in complex surgery. J Public Health. 2006;28(4):375-378.

25. Canda AE, Sokmen S, Terzi C, et al. Complications and toxicities after cytoreductive surgery and hyperthermic intraperitoneal chemotherapy. Ann Surg Oncol. 2013;20(4):1082-1087.

\section{Publish your work in this journal}

Therapeutics and Clinical Risk Management is an international, peerreviewed journal of clinical therapeutics and risk management, focusing on concise rapid reporting of clinical studies in all therapeutic areas, outcomes, safety, and programs for the effective, safe, and sustained use of medicines. This journal is indexed on PubMed Central, CAS,
EMBase, Scopus and the Elsevier Bibliographic databases. The manuscript management system is completely online and includes a very quick and fair peer-review system, which is all easy to use. Visit http://www.dovepress.com/testimonials.php to read real quotes from published authors.

Submit your manuscript here: http://www.dovepress.com/therapeutics-and-clinical-risk-management-journal 